\title{
Future Radiometer Systems for Earth Remote Sensing
}

\author{
William J. Wilson and Eni G. Njoku \\ Bldg 168-327, 4800 Oak Grove Drive \\ Jet Propulsion Laboratory \\ California Institute of Technology \\ Pasadena, CA 91109
}

Abstract--This paper will describe a new exciting concept for using microwave systems for Earth remote sensing. This concept will use a 6-m diameter mesh deployable antenna with active and passive systems to provide moderate spatial resolution images at $L$ and S-band microwave frequencies.

\section{INTRODUCTION}

Microwave radiometry and scatterometry are preferred techniques for many Earth remote sensing applications from space, particularly measurements of soil moisture, snow cover, sea ice extent and motion, sea surface salinity, sea surface temperature, and sea surface wind speed and direction. Achieving a small spatial resolution for these measurements from space requires much larger effective apertures at microwave frequencies than at optical and infrared frequencies. Techniques and technology options for achieving the desired microwave system performance (spatial resolution and accuracy) have been studied using low-mass mesh deployable antennas. The focus was on the soil moisture and ocean salinity science applications since they require low microwave frequencies which drive the requirement for large antennas, and cannot at present be measured adequately from space.
For soil moisture and salinity sensing, the desired frequencies are in the range from 1 to $3 \mathrm{GHz}$, and our study focused on this frequency range. However, the concept and technologies evaluated are applicable to all the science parameters listed above, which can be sensed by using additional measurement channels at frequencies up to 14 GHz. An optimal system configuration has been developed for a spaceborne mission, based on science requirements for accuracy, stability, spatial resolution, and spatial and temporal coverage, while also having the potential to meet the requirements for a lowcost space mission with a short development cycle. The system characteristics of this concept are given in Table 1.

\section{DESIGN CONCEPT}

In the present design, the radiometers operate at two frequencies (1.41 and 2.69 $\mathrm{GHz})$ and the radar at a single frequency $(1.26$ $\mathrm{GHz}$ ) both with dual polarization. The 1.41 $\mathrm{GHz}$ frequency is required to obtain adequate sensitivity to ocean salinity and soil moisture. The $2.69 \mathrm{GHz}$ frequency, and dual polarization, are required to provide corrections for surface roughness and surface temperature over ocean, and vegetation cover over land. The $1.26 \mathrm{GHz}$ radar channel will provide corrections, with higher sensitivity, to ocean roughness and vegetation land cover 
Table 1. System Characteristics

\begin{tabular}{|l|l|}
\hline Radiometer Frequencies & $\mathrm{I} .41$ and $2.69 \mathrm{GHz}$ \\
\hline Radiometer Polarizations & $\mathrm{V}, \mathrm{H}$ \\
\hline Antenna Aperture & $6 \mathrm{~m}$ \\
\hline Antenna Beamwidth and Beam Efficiency & $2.6^{\circ},>90 \%$ \\
\hline Radar Frequency & $1.26 \mathrm{GHz}$ \\
\hline Radar Polarization Modes & $\mathrm{VV}, \mathrm{HH}, \mathrm{VH}$ \\
\hline Orbit altitude & $600 \mathrm{~km}$ \\
\hline Incidence Angle & $40^{\circ}$ \\
\hline Footprint Size & $35 \times 46 \mathrm{~km}$ \\
\hline Swath Width & $900 \mathrm{~km}$ \\
\hline No. Antenna Beams per frequency & 2 \\
\hline Rotation Rate & $6.1 \mathrm{rpm}$ \\
\hline RMS Noise per pixel (B = 100 MHz) & $0.1 \mathrm{~K}$ \\
\hline Radiometer Absolute Calibration Accuracy & $1.0 \mathrm{~K}$ \\
\hline Radar Calibration Accuracy & $1 \mathrm{~dB}$ \\
\hline Radar Transmit Power and Duty Cycle & $200 \mathrm{Watts,10 \%}$ \\
\hline Data Rate & $20 \mathrm{Kbits} / \mathrm{sec}$ \\
\hline Power & $300 \mathrm{Watts}$ \\
\hline
\end{tabular}

- Over ocean, and averaging forward and rear pixels. Over land, the bandwidths are reduced to $25 \mathrm{MHz}$ and $10 \mathrm{MHz}$ at 1.41 and $2.69 \mathrm{GHz}$, respectively, to minimize radio-frequency interference.

than can be provided by radiometric channels alone.

The antenna concept is a 6-m-diameter rotating, offset-fed, parabolic mesh reflector, with a cluster of two multi-frequency feedhorns. The combined antenna and feed system rotates about the vertical axis with antenna beams offset at a constant angle from nadir, providing a conical scan and wide swath for frequent global sampling. One possible configuration, and the conical scan pattern are shown in Figure 1. As the spacecraft moves, the 3-dB antenna footprints provide complete overlap coverage along track and across the swath.

Each of the feedhorns is shared between the 1.41 and $2.69 \mathrm{GHz}$ radiometers and the $1.26 \mathrm{GHz}$ radar as shown in Figure 2. The radiometer calibration will be done using a noise diode coupled in after the feedhorns, and the radar will be calibrated with a coupling loop. If higher frequency channels 


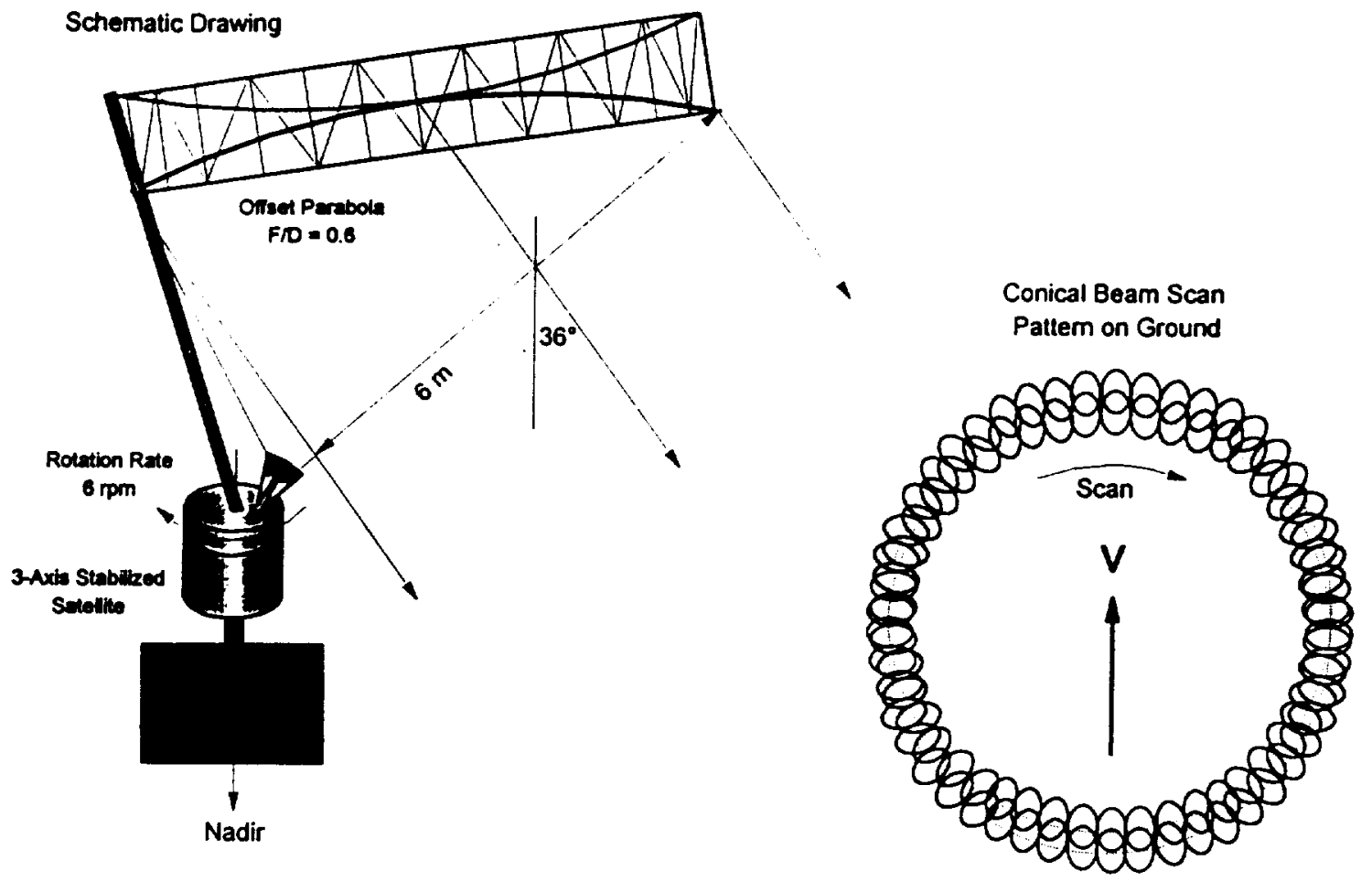

Figure 1. Soil Moisture and Salinity Instrument Concept

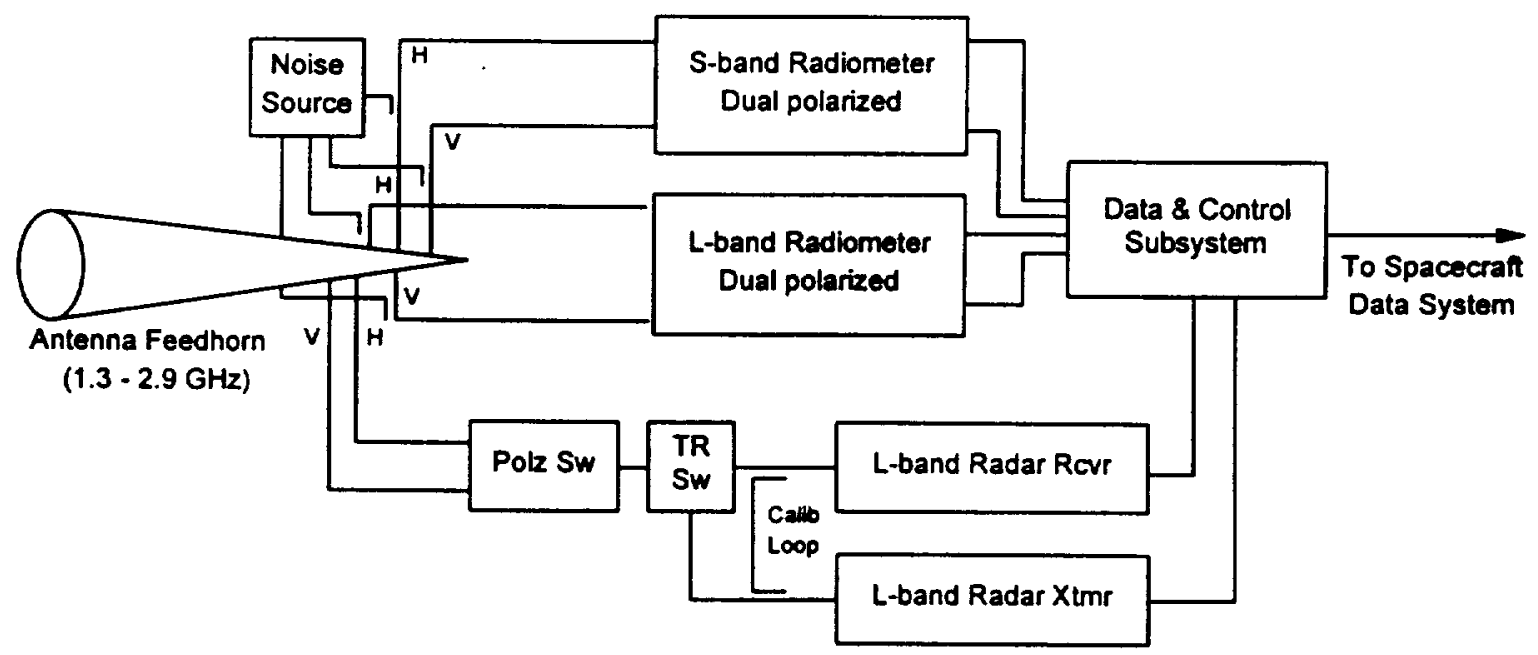

Figure 2. Block Diagram of Microwave Electronics System 
are included, then the smaller feedhorns will be clustered around the lower frequency feeds. The two feedhorns provide two separate beams, allowing the reflector to rotate at the relatively moderate rate of $6 \mathrm{rpm}$.

A momentum wheel in the spacecraft compensates for the momentum of the spinning assembly. Under-illumination of the main reflector at $2.69 \mathrm{GHz}$ will provide nearly equal beamwidths at 1.41 and $2.69 \mathrm{GHz}$.

There are a number of different structural concepts for mesh deployable antennas. These include the "radial rib" and "perimeter truss" concepts. The perimeter truss is a relatively new concept, based on a toroidal-shaped structure that supports the mesh reflector and its surface-shaping network of cables and ties at their outer perimeter. A number of mesh deployable antennas have been launched in previous space missions, with TDRSS being the best known example. Mesh-deployable antennas in the 12-m-diameter size range, using both radial rib and perimeter truss designs will be launched in 1999 and 2000 for geostationary communication satellite applications [1].

\section{SUMMARY}

In this paper a system configuration has been presented for a multi-channel (passive and active) microwave spaceborne sensor operating in the $1-3 \mathrm{GHz}$ frequency range. It is proposed to use a rotating 6-m diameter offset parabolic mesh antenna. Antennas of this type have flown in space before and new ones are planned. Thus, this concept has a long successful history and provides confidence for future Earth remote sensing missions.

\section{ACKNOWLEDGMENTS}

This research was carried out by the Jet Propulsion Laboratory, California Institute of Technology, under contracts with the National Aeronautics and Space Administration.

\section{REFERENCE}

1. B. Miller, "Satellites free the mobile phone," IEEE Spectrum, vol. 35, pp. 26-35, 1998. 